\title{
The Layer 7 Cortical Interface: A Scalable and Minimally Invasive Brain-Computer Interface Platform
}

Elton $\mathrm{Ho}^{1}{ }^{*}$, Mark Hettick ${ }^{1}$, Demetrios Papageorgiou ${ }^{1}$, Adam J. Poole ${ }^{1}$, Manuel Monge ${ }^{1}$, Maria Vomero $^{1}$, Kate R. Gelman ${ }^{1}$, Timothy Hanson ${ }^{1}$, Vanessa Tolosa ${ }^{1}$, Michael Mager ${ }^{1}$, Benjamin I. Rapoport $^{1+}$

${ }^{1}$ Precision Neuroscience Corporation, New York, New York, and San Francisco, California, United States of America

* These authors contributed equally to this work

+ Corresponding author

\begin{abstract}
Progress toward the development of brain-computer interfaces has signaled the potential to restore, replace, or augment lost or impaired neurological function in a variety of disease states. Existing brain-computer interfaces rely on invasive surgical procedures or brain-penetrating electrodes, which limit addressable applications of the technology and the number of eligible patients. Here we describe a novel approach to constructing a neural interface, comprising conformable thin-film electrode arrays and a minimally invasive surgical delivery system that together facilitate communication with large portions of the cortical surface in bidirectional fashion (enabling both recording and stimulation). We demonstrate the safety and feasibility of rapidly delivering reversible implants containing over 2,000 microelectrodes to multiple functional regions in both hemispheres of the Göttingen minipig brain simultaneously, without requiring a craniotomy, at an effective insertion rate faster than $40 \mathrm{~ms}$ per channel, without damaging the cortical surface. We further demonstrate the performance of this system for high-density neural recording, focal cortical stimulation, and accurate neural decoding. Such a system promises to accelerate efforts to better decode and encode neural signals, and to expand the patient population that could benefit from neural interface technology.
\end{abstract}




\section{Introduction}

Brain-computer interfaces have shown promise as systems for restoring, replacing, and augmenting lost or impaired neurological function in a variety of contexts, including paralysis from stroke and spinal cord injury, blindness, and some forms of cognitive impairment ${ }^{1-11}$. Multiple innovations over the past several decades have contributed to the potential of these neural interfaces, including advances in the areas of applied neuroscience and multichannel electrophysiology ${ }^{12-21}$, mathematical and computational approaches to neural decoding ${ }^{17,22-28}$, power-efficient custom electronics and the development of application-specific integrated circuits $^{29-41}$, as well as materials science and device packaging ${ }^{42-49}$. Nevertheless, the practical impact of such systems remains limited, with only a small number of patients worldwide having received highly customized interfaces through clinical trials ${ }^{50-52}$.

In order to achieve meaningful clinical impact on the large populations of patients who stand to benefit from brain-computer interface technologies, surgical procedures involved in implanting neural interfaces should be minimally invasive and reversible, and avoid damaging neural tissue. Advanced brain-computer interfaces require collection and processing of large amounts of neural data, potentially spanning multiple brain regions. As a result, high-density microelectrode arrays have been replacing more traditional macroelectrode arrays, offering smaller features and improved spatial resolution ${ }^{53-58}$. Systems for clinical use should also demonstrate a high degree of scalability in terms of channel count and speed of implantation. Tissue damage and total procedural time cannot be allowed to increase in proportion to channel number, as contemporary channel counts, already reaching the many thousands, will likely increase by orders of magnitude with further progress in this field.

Microelectrode arrays that penetrate the brain have facilitated high-spatial-resolution recordings for brain-computer interfaces, but at the cost of invasiveness and tissue damage that scale with the number of implanted electrodes ${ }^{20,59-62}$. It is not yet clear whether approaches involving softer penetrating electrodes offer a substantially different tradeoff ${ }^{20,59}$. For this reason, non-penetrating cortical surface microelectrodes represent a potentially attractive alternative and form the basis of the system described here ${ }^{53,63}$. In practice, electrocorticography (ECoG) has already facilitated capture of high quality signals for effective use in brain-computer interfaces in several applications, including motor and speech neural prostheses ${ }^{7,22,24,56,64-70}$. Higher-spatialresolution micro-electrocorticography $(\mu \mathrm{ECoG})$ therefore represents a promising combination of minimal invasiveness and improved signal quality.

Here we demonstrate a modular and highly scalable system of conformable thin-film microelectrodes designed for rapid, minimally invasive deployment on the cortical surface. We describe $\mu \mathrm{ECoG}$ array fabrication and minimally invasive insertion techniques. We demonstrate the practicality and in vivo performance of the system by implanting thousands of electrodes simultaneously in multiple regions of the neocortex in both hemispheres, including areas related to vision and somatosensory function. We further demonstrate the use of these arrays for electrophysiologic functions required of contemporary brain-computer interfaces, including neural recording, cortical stimulation, and decoding. The platform is intended to facilitate human clinical use of brain-computer interface technology by delivering the microelectrode numbers and spatial densities required for advanced, high-performance brain-computer interface applications in a safe and time-efficient manner that is compatible with proven and reliable neurosurgical techniques. 


\section{Results}

System Overview. The system as configured for in vivo neural recording and stimulation comprises a modular set of thin-film microelectrode arrays designed for rapid, minimally invasive subdural implantation using a novel "cranial micro-slit" technique. Each microelectrode array comprises 529 electrodes ranging in diameter from 20 to $200 \mu \mathrm{m}$ and is connected to a customized hardware interface. After subdural array implantation the interconnecting cable of each microelectrode array module passes through a dural incision and a cranial micro-slit incision, is tunneled under the scalp as needed, and is connected to an individual head stage. The overall system configuration is illustrated in Figure 1.

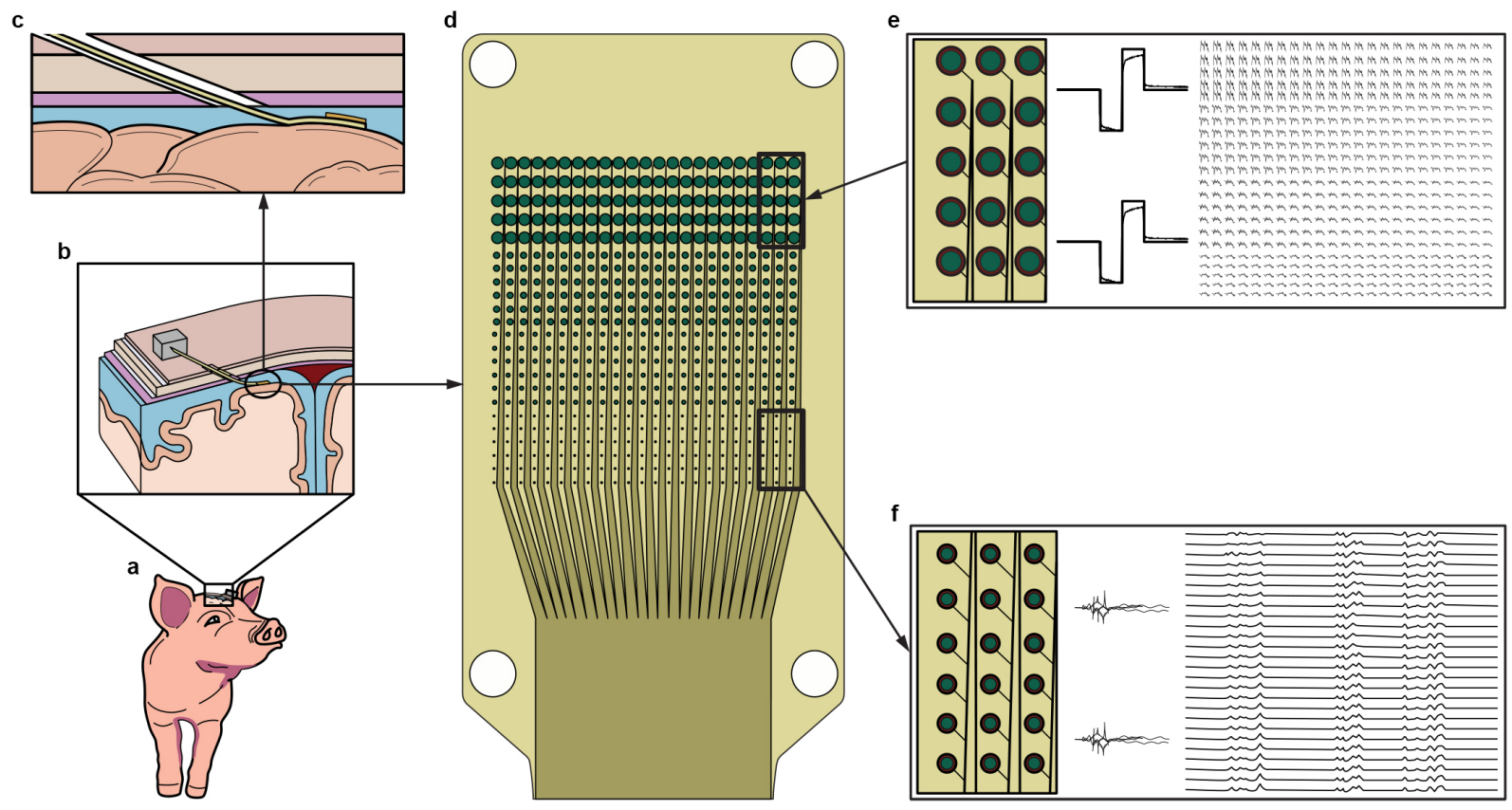

Figure 1. System Overview. This schematic illustration shows how neural activity is acquired from and stimulated by the Layer 7 Cortical Interface. (a) A Göttingen minipig undergoes cranial micro-slit implantation ( $b$ and $c$ ) of a set of subdural microelectrocorticography arrays comprising a total of $>2000$ microelectrodes, in modules containing 529 channels each (d). In the anatomic schematics (b and c) the subdural space is shown in blue, dura in purple, skull in beige. The outermost layer shown represents the scalp. (e) A group of $200 \mu \mathrm{m}$ electrodes is shown in detail together with example stimulation waveforms, and the resulting post-stimulus activity recorded over the entire array. (f) A group of $20 \mu \mathrm{m}$ microelectrodes is shown in detail together with example traces from recorded neural activity. The microelectrode arrays provide multichannel data that is used in a variety of electrophysiologic paradigms to perform neural recording of both spontaneous and stimulus-evoked neural activity as well as decoding and focal stimulation of neural activity across a variety of functional brain regions.

Electrode Array Characterization. All microelectrode arrays were thoroughly characterized prior to insertion. Prior to assembly, bonded microelectrode array-interposer assemblies were 
optically inspected in bond, cable, and electrode areas. Impedance spectra of the platinum electrodes (shown in the photographs and photomicrographs of Figures $2 \mathrm{a}$ and $2 \mathrm{~b}$, respectively) verified direct correspondence between electrode area and impedance magnitude (Figure $2 \mathrm{~b}$ ). Process yield was evaluated with respect to electrode connectivity and was confirmed to be $>93 \%$ after optimization, by impedance characterization of 4,232 electrodes from eight arrays. A set of electrodes of each size was also characterized electrochemically by cyclic voltammetry (CV) and electrochemical impedance spectroscopy (EIS). EIS measurements for each electrode size display typical positive shifts in cutoff frequencies with decreases in electrode size, and impedance magnitude at $1 \mathrm{kHz}$ also follows the expected upward trend with decreasing electrode size (Figure b). In vivo electrode impedance mapping results confirm high electrode yield and impedance uniformity (Figure 2b, inset). As expected, electrode impedance demonstrates a predictable dependence on electrode surface area, ranging from an average of $497 \mathrm{k} \Omega$ for $20 \mu \mathrm{m}$ electrodes to $32 \mathrm{k} \Omega$ for $200 \mu \mathrm{m}$ electrodes. A key factor for our system is also the robustness of arrays during the implantation process, as demonstrated by the ratio of impedance before and after implantation showing little change across the array (Figure 2c).
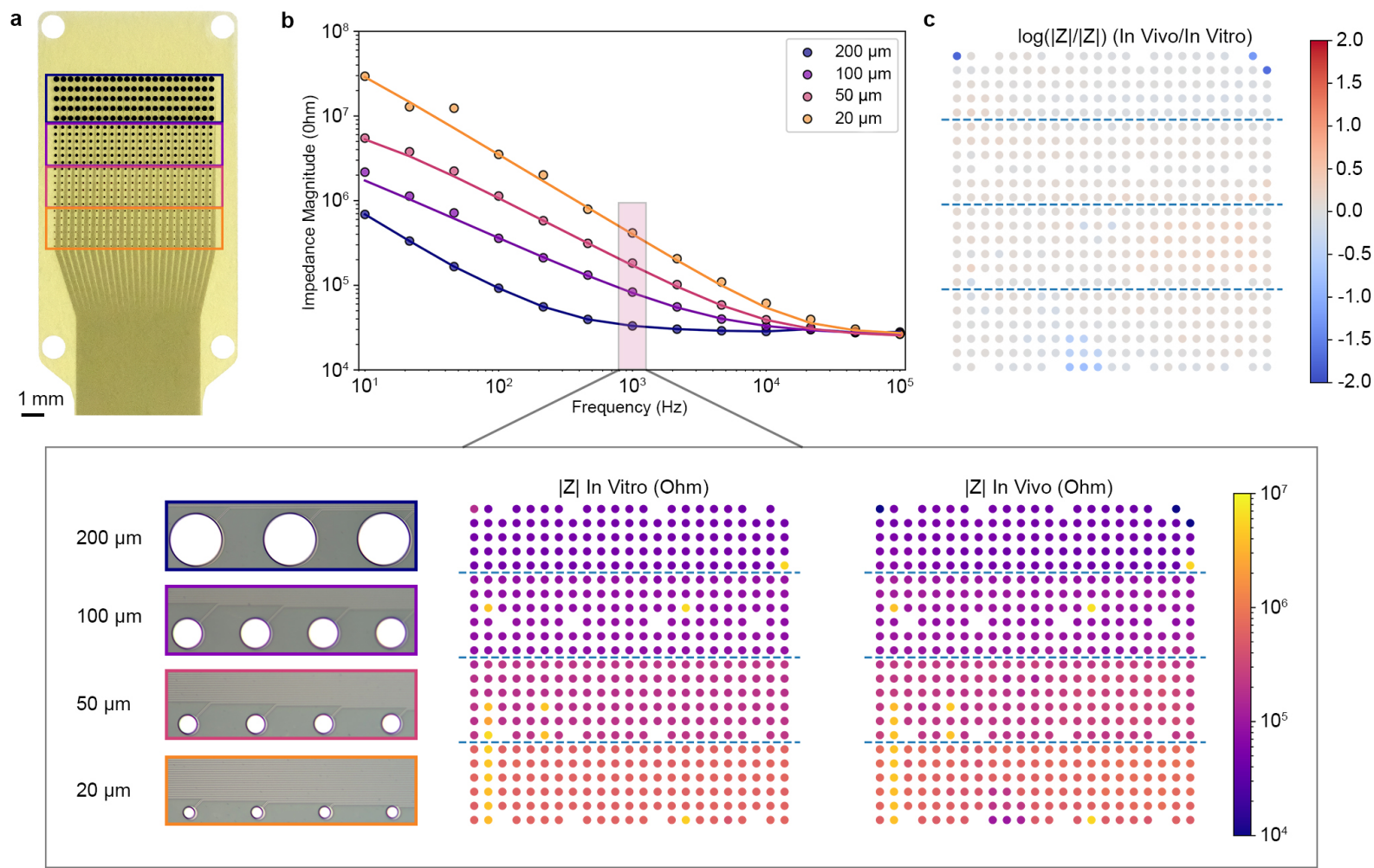

Figure 2. Electrode Characterization. (a) Photograph of a single array with 200, 100, 50, and $20 \mu \mathrm{m}$ electrodes outlined by blue, purple, pink, and orange boxes, respectively. (b) In vitro electrochemical impedance spectroscopy (EIS) spectra for each electrode size, with equivalent circuit fits based on a Randles circuit. (Inset) In vitro (left) and in vivo (right) impedance maps of recording channels over a complete 529-channel array, measured at 1 $\mathrm{kHz}$, with microscope images for each electrode size included as references. Stimulation and ground channels are shown as gaps in the plots. (c) Map of the ratio between in vivo and in vitro impedance at $1 \mathrm{kHz}$, displaying minimal change across most of the array following implantation. 
Minimal Invasiveness and Speed. We have demonstrated the safety and feasibility of inserting our high-density microelectrode arrays using a novel, minimally invasive "cranial micro-slit" technique (Figure 3). The procedure employs a precision oscillating blade to make 400-micronwide incisions in the skull at approach angles tangential to the cortical surface, facilitating subdural insertion of our thin-film arrays without requiring a burr hole or craniotomy. To validate the procedure we performed increasing numbers of cranial micro-slit insertions in a series of three Göttingen minipigs (1, 2, and 3 slits, respectively). The insertion mechanism is shown in Figure 3a. Insertion time following cranial and dural incisions was 20-40 s. For a single array of 529 microelectrodes, this corresponds to an average insertion rate of $<40-80 \mathrm{~ms}$ per electrode over 0.48 $\mathrm{cm}^{2}$ of cortical surface area.

Safety and Reversibility. Four insertion sites were inspected by craniectomies immediately postmortem, following euthanasia, to assess the cortical surface for evidence of tissue damage following array removal ("reversal" of implantation). An example image of the cortical surface after array removal and postmortem craniectomy is shown in Figure 3c. The pia remained intact following array placement and removal, and we found no macroscopic evidence of tissue disruption at the cortical surface corresponding to array placement. 

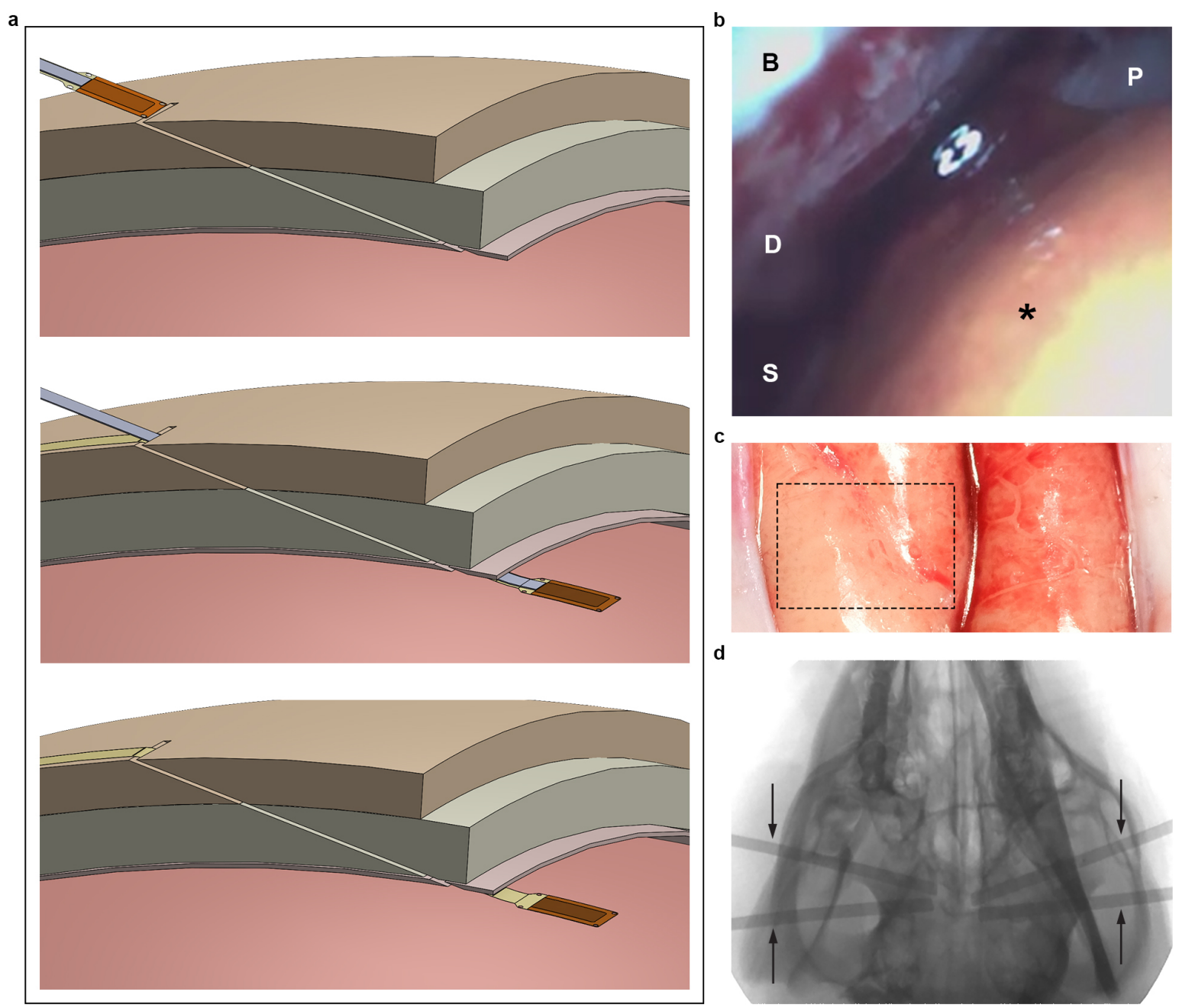

Figure 3. Insertion Technique. (a) The "cranial micro-slit" insertion technique is illustrated schematically, showing guidance of the thin-film-based array into the subdural space. A scalp incision is made, followed by a cranial incision, both at an angle tangent to the cortical surface in the location targeted for array placement; the dura is then coagulated and incised (not shown). The trajectory of these aligned incisions is shown, together with an electrode array mounted on a stiffening shim for subdural implantation through the cranial slit. The shim inserts into a polyimide pocket on the back of the array (top). The array-shim assembly is inserted into the subdural space (center). The shim is then removed, leaving the array in place (bottom). (b) Intraoperative endoscopic view immediately following subdural placement of one thin-film array, after shim removal, showing the electrode tail and traces entering the subdural space through a cranial slit (B, cut surface of skull seen from within the cranial micro-slit; D, undersurface of the dura; S, subdural space; $\mathrm{P}$, pia of the cortical surface; the asterisk denotes the tail of the array, $5.49 \mathrm{~mm}$ in width, as seen from within the micro-slit). (c) View of the intact cortical surface postmortem corresponding to the region of subdural array placement. The dotted outline indicates the position of the array before removal (primary somatosensory cortex). (d) Intraoperative fluoroscopic image (dorsoventral projection) showing four 529-electrode arrays in place, two over each hemisphere. Black arrows indicate the radiopaque shims used to position the arrays. 
Modularity and Scalability. The fabricated arrays facilitate alignment and modular assembly, as shown in Figure 4, so that multiple 529-electrode modules can be joined to yield larger constructs covering larger portions of the cortical surface at the same density, without significantly increasing the complexity, risk, or time required for array insertion. It is also possible to insert multiple arrays through a single slit. We performed in vivo insertions of doubly connected modules $(1,058$ channels over $0.96 \mathrm{~cm}^{2}$ of cortical surface area), and in vitro insertions of quadruply connected modules $\left(2,116\right.$ channels each covering $1.92 \mathrm{~cm}^{2}$ of surface area). We note that use of a doubly connected module halves the effective average insertion rate, and in this manner have achieved effective speeds as fast as $<20 \mathrm{~ms}$ per electrode in vivo.

We have also demonstrated the ability to interface with multiple anatomic and functional areas of the neocortex simultaneously in vivo, performing bilateral insertions over somatosensory cortex, as shown fluoroscopically in Figure $3 \mathrm{~d}$ and confirmed by somatosensory evoked potentials (Figure 5e), and additional insertions over visual cortex, as indicated schematically in Figure 5a and confirmed by visual evoked potentials (Figure 5f).

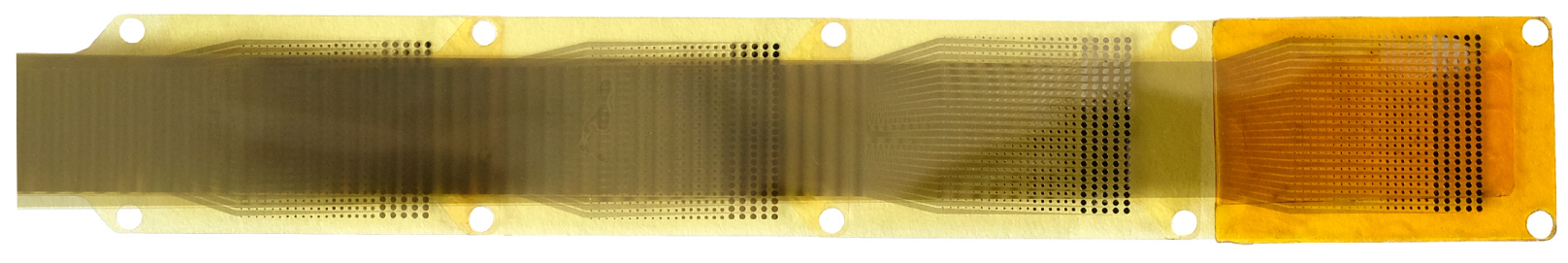

Figure 4. Modularity and Scalability. Modular configurations of multiple 529-channel arrays can be constructed. Shown here is a quadruply-connected assembly of four 529electrode modules with a single pocket, for simultaneous implantation of 2116 microelectrodes over $1.92 \mathrm{~cm}^{2}$ of cortical surface area. Scale bar: $1 \mathrm{~mm}$.

Neural Recording. Implanted arrays were used for multichannel neural recording using several paradigms, capturing both spontaneous cortical electrographic activity (Figure 5b) as well as somatosensory evoked potentials (Figure 5e) and visual evoked potentials (Figure 5f).

During recording of spontaneous cortical activity, multiple 3-minute epochs were captured and analyzed. Electrocorticograms were obtained and reviewed in time and frequency domains. Representative time traces and spectrograms are shown in Figures $5 \mathrm{~b}-\mathrm{d}$.

Evoked potentials were obtained across multiple arrays and multiple functional regions of the brain in the same session. Robust array-based somatosensory evoked potentials were demonstrated in arrays positioned over somatosensory cortex, corresponding to tactile stimulation of the hindlimbs (Figure 5e), and visual evoked potentials were also obtained in arrays positioned over visual cortex following time-synchronized photostimulation of the retina (Figure 5f). 
b

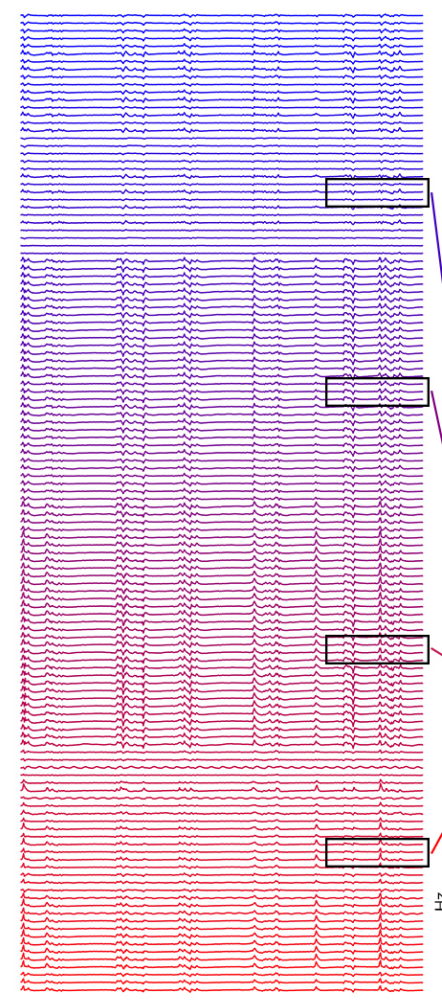

a

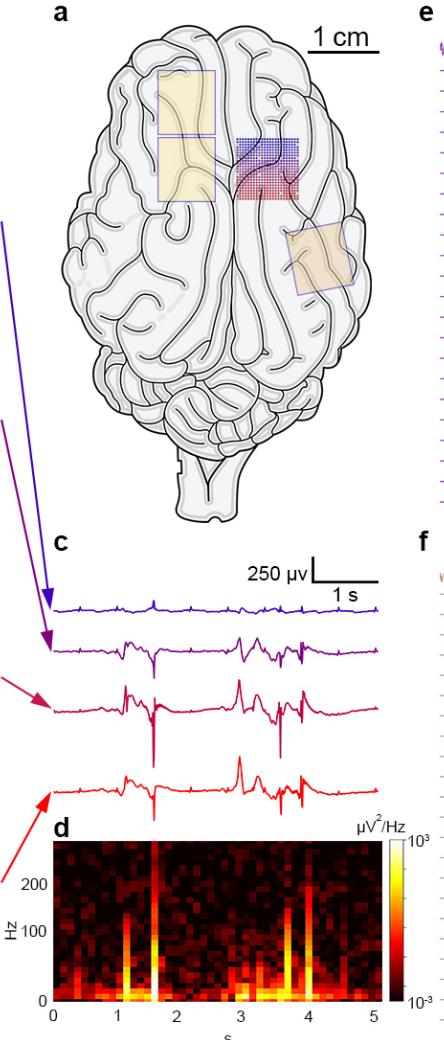

e

f
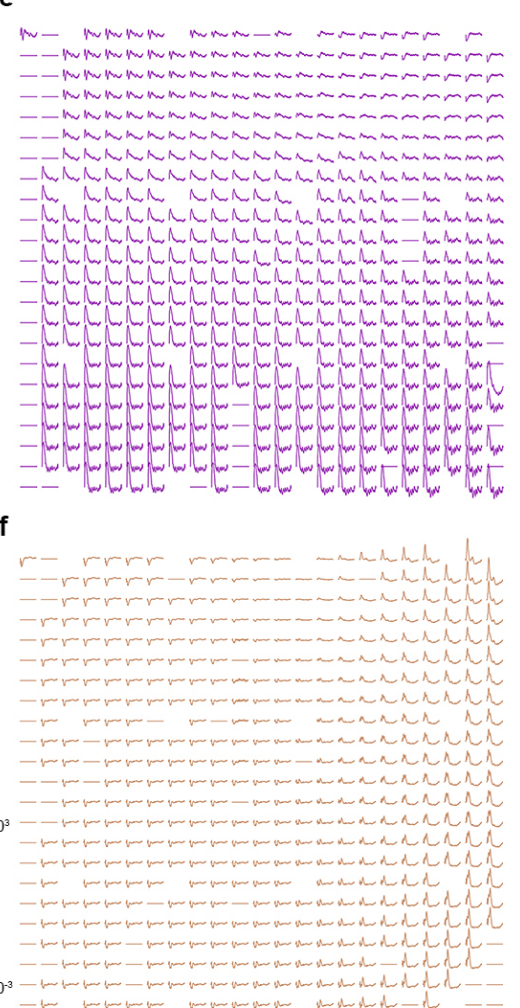

Figure 5. Neural Recordings. (a) Schematic of the Göttingen minipig brain showing color-coded areas corresponding to placement of subdural microelectrode arrays. (b) Recording of spontaneous neural activity from somatosensory cortex in a sample of channels from the highlighted 529-channel module. (c) Example recording traces, and (d) example spectrogram corresponding to the highlighted channels from an array over right somatosensory cortex. (e) Somatosensory evoked potentials corresponding to tactile stimulation of the left hindlimb. Traces span $1 \mathrm{~s}$ and the maximum peak-to-peak signal is approximately $80 \mu \mathrm{V}$. (f) Visual evoked potentials from photostimulation of the left eye.

Neural Decoding. Insights into the utility of this system for neural decoding are provided by both spontaneous activity and evoked potentials. Figure 6 illustrates the degree to which spontaneous neural activity, as recorded from reference sites sampled from across a given array, is correlated with activity simultaneously recorded from other sites on the same array. The degree of correlation decreases with distance (Figure 6a) and with increasing frequency (Figure 6b). Importantly, even closely spaced electrodes exhibit incompletely correlated activity, particularly at higher frequencies. For example, broadband $r^{2}$ is in the range of 0.8 at $300 \mu \mathrm{m}$ spacing for $20 \mu \mathrm{m}$ electrodes. These properties of the sampled electrocorticographic activity, together with the ability to record robust evoked potential responses across hundreds of channels (Figure 5e-f), facilitate straightforward neural decoding, as summarized in Figure $6 \mathrm{c}-\mathrm{d}$ for visual stimulation. We used an unweighted template-matching algorithm as the basis for distinguishing stimulation of the left or right eye from absence of stimulation (free recording). Multichannel decoding using the large number of electrodes available from the entire array was more robust than decoding using any single electrode, with accuracies reaching $73 \%$ on the left and $67 \%$ on the right in off-line decoding. 
a

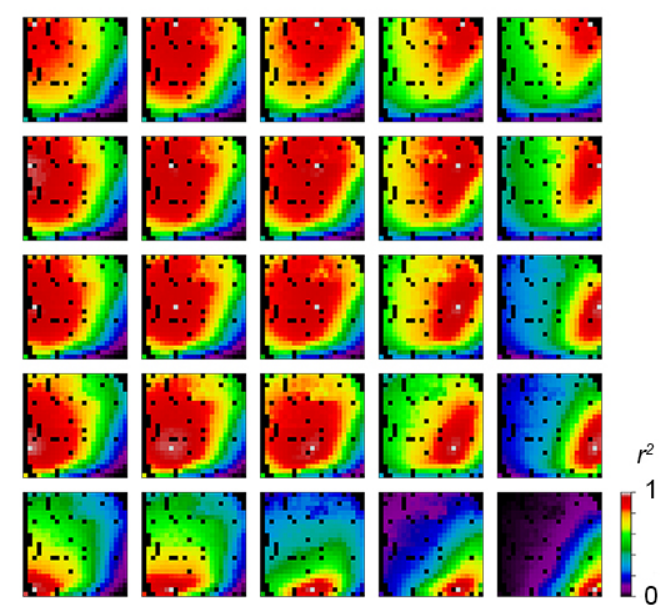

C

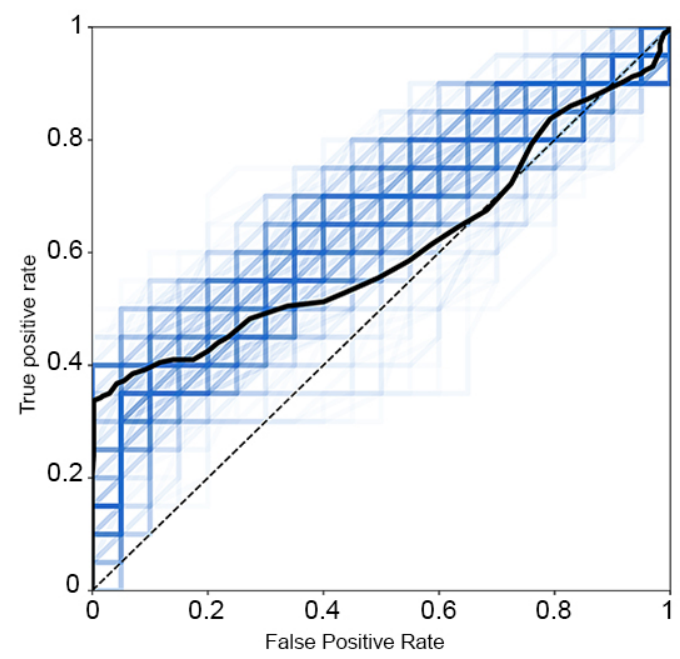

b

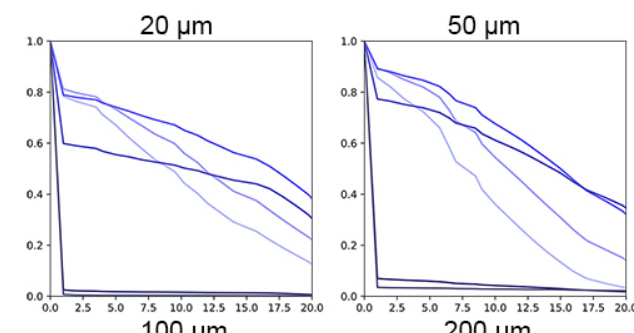

$100 \mu \mathrm{m}$
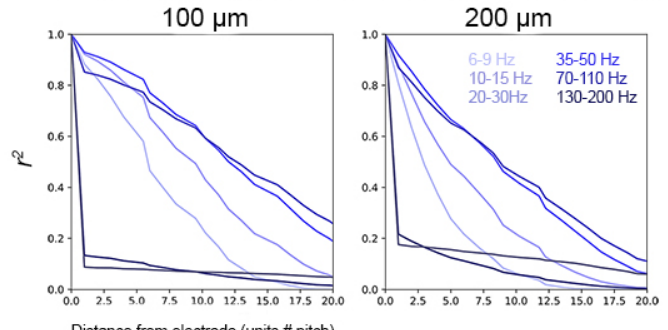

Distance from electrode (units \# pitch)

d

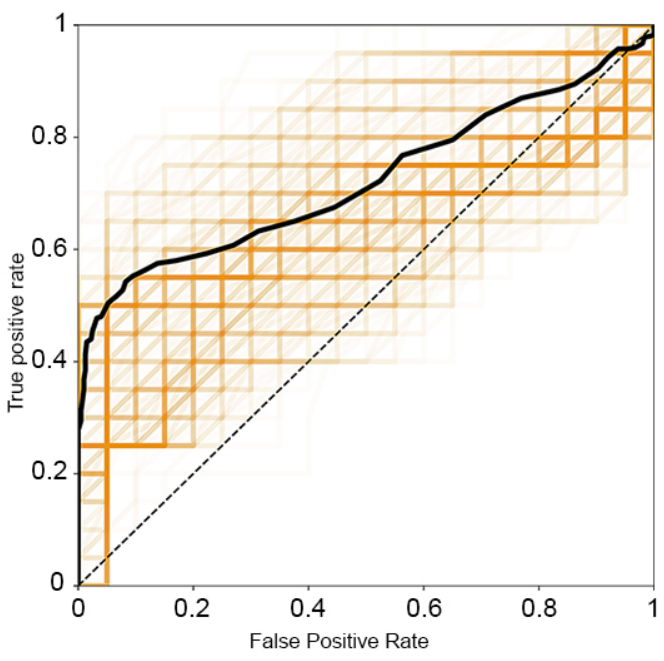

Figure 6. Decoding. The degree to which closely spaced microelectrodes across a single 529-channel array record correlated or uncorrelated information is relevant to neural decoding and is illustrated here in several ways. (a) Correlation map of the Pearson correlation coefficient $r^{2}$ computed for signals recorded during spontaneous cortical activity, with correlations across the entire array in each sub-plot referenced to one of 25 electrodes (grey dots) distributed evenly over the array, as represented by the array of subplots. (b) The dependence of correlation on electrode separation as a function of electrode size and spectral band. Characteristics of the accuracy and robustness of neural decoding from right visual cortex are shown in (c) and (d). Shown are the receiver operating characteristic (ROC) curves for decoding visual stimulation of the left (c) or right (d) eye. Colored traces represent the ROC curves from template matching individual channels, while the black trace represents decoding performance obtained by integrating information from all channels into a collective template (averaged in Monte Carlo fashion over 20 shuffles of the training and testing datasets). 
Neural Stimulation. The electrode array is capable of bidirectional function, with every electrode able to perform either recording or stimulation. As configured for the described set of in vivo experiments, 16 electrodes per array were designated for use in cortical stimulation. Safe stimulation thresholds were determined in vitro for each electrode type. Briefly, the water window was determined by CV measurement to be approximately $1.85 \mathrm{~V}$ (with a $0.65 \mathrm{~V}$ cathodic and 1.2 $\mathrm{V}$ anodic limit), and biphasic pulses were applied at $50 \mathrm{~Hz}$ to a test electrode within the range of anticipated stimulation parameters to confirm safe stimulation (Figure 7a). A total of 50,000 pulses of $10 \mathrm{nC}$ per phase and 100,000 pulses of $20 \mathrm{nC}$ per phase (50 and $100 \mu \mathrm{A}$ respectively with a 200 $\mu$ s pulse width) were found to cause no change in impedance on the test electrode, as well as polarization potentials within the water window, so stimulation currents in this range were determined to be acceptable for the 30 pulses per electrode used in vivo. Cortical stimulation was performed in vivo, with a $200 \mu \mathrm{m}$ electrode used for stimulation in each trial, and the remaining sites on the same array, as well as all sites on adjacent arrays, were used for recording.

After characterizing a safe operating regime for cortical stimulation, we performed focal stimulation of the visual cortex using the paradigm described, while recording stimulus-evoked cortical activity (Figure 7). Cortical stimulation evoked an increase in high-gamma-band power across the surrounding region monitored by the array, lasting approximately $1 \mathrm{~s}$. The most highly activated region formed an annulus of radius approximately $1 \mathrm{~mm}$ around, but not immediately surrounding the stimulating electrode. Regions farther from the annulus exhibited a transient reduction in cortical activation during this period. No induced saccades were observed during stimulation of the visual cortex. 
a

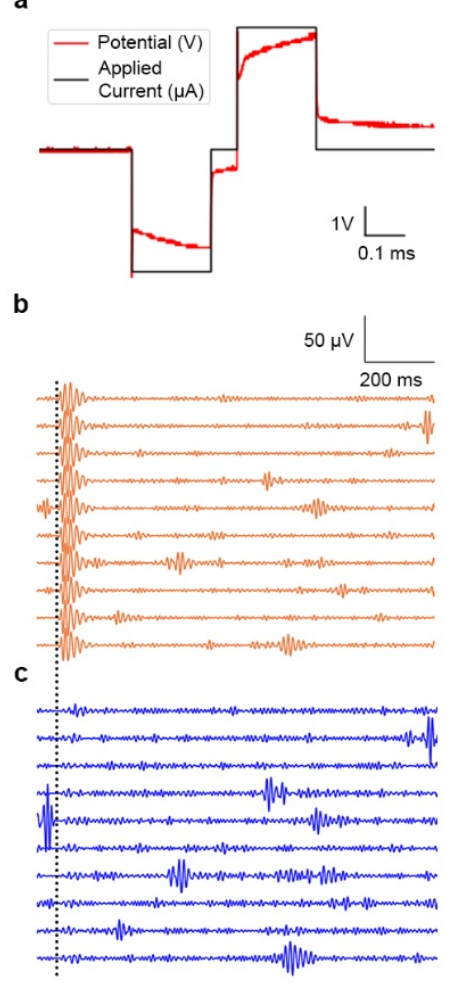

d

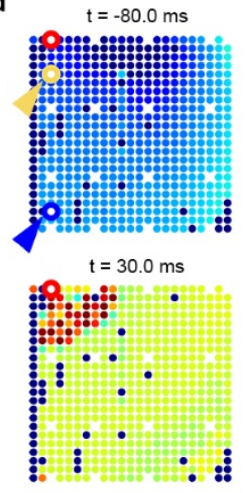

$\mathrm{t}=200.0 \mathrm{~ms}$

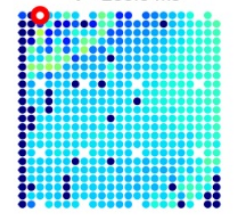

$t=495.0 \mathrm{~ms}$

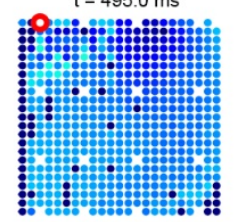

$t=0.0 \mathrm{~ms}$

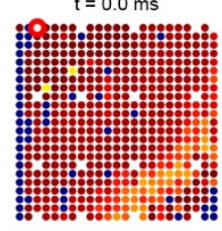

$t=50.0 \mathrm{~ms}$

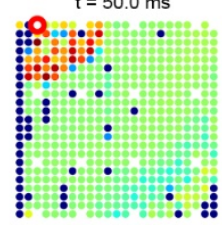

$t=270.0 \mathrm{~ms}$

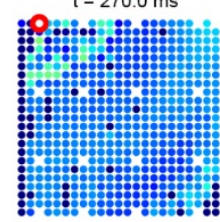

$t=620.0 \mathrm{~ms}$

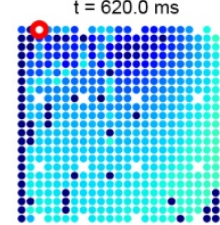

$t=20.0 \mathrm{~ms}$

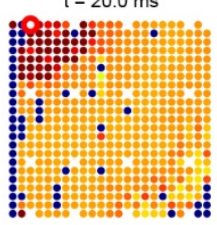

$t=-80.0 \mathrm{~ms}$

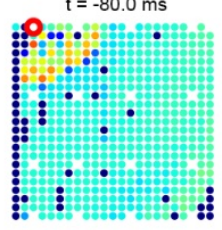

$\mathrm{t}=320.0 \mathrm{~ms}$

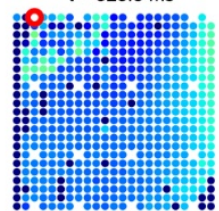

$t=720.0 \mathrm{~ms}$

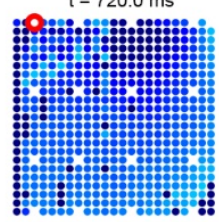

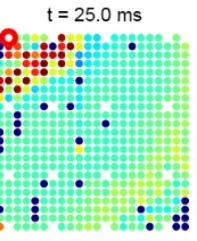

$t=140.0 \mathrm{~ms}$

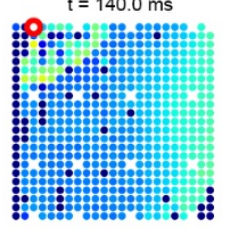

$t=370.0 \mathrm{~ms}$

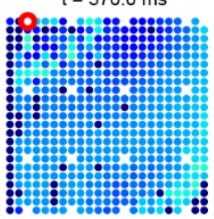

$\mathrm{t}=820.0 \mathrm{~ms} \quad \mu \mathrm{V}^{2} / \mathrm{Hz}$

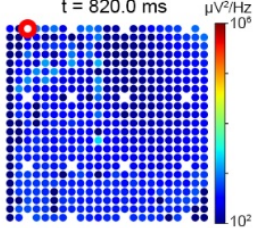

Figure 7. Cortical Stimulation. (a) Stimulation waveform used for in vitro confirmation of safe polarization potential, with the $100 \mu \mathrm{A}$ current overlaid on the waveform for reference. In vivo applied current waveforms used the same applied current but without the interphase delay used for identifying polarization potential. (b) Example traces for an electrode near the stimulation electrode (red ring) band-pass filtered for 70-150 Hz (highgamma band) from 10 stimulation trials recording from one microelectrode near the stimulation site (orange ring). These trials reveal increased cortical local field activity immediately following cortical stimulation for regions near the stimulating electrode. Dotted vertical line indicates the stimulus. (c) Corresponding traces for an electrode farther from the stimulus (blue ring). While still being responsive to stimulation, more distant regions exhibit less evoked activity. (d) Time progression of high-gamma-band power across the array of microelectrodes. Stimulation starts at $t=0 \mu \mathrm{s}$ and ends at $t=400 \mu \mathrm{s}$. 


\section{Discussion}

Here we have described a modular and highly scalable brain-computer interface platform capable of rapid, minimally invasive surgical deployment over multiple large areas of the cortical surface in a reversible and atraumatic manner. The system provides high channel counts and high spatial densities of electrode coverage, as well as bidirectional functionality (both neural recording and neural stimulation). The platform is based on thin-film microelectrode arrays that conform to the shape of the cortical surface. The microelectrode arrays are designed to be inserted into the subdural space through 400-micron-wide skull incisions made using a precision oscillating blade, guided by real-time imaging and fiberoptic endoscopy, at a rate of more than 1,000 electrodes per minute, and to provide 1,000-fold higher electrode density than standard cortical grids. The platform is designed to deliver the large microelectrode numbers and high spatial densities required to facilitate confident adoption and routine clinical use of advanced, high-performance braincomputer interface technologies.

We believe that one important set of design constraints for clinically useful neural interfaces relates to the ability to implant large numbers of microelectrodes at high spatial densities, without damaging the brain, in a manner that is reversible and that can be performed rapidly, relatively painlessly, and ultimately without the need for general anesthesia. The Layer 7 Cortical Interface system described here is designed to satisfy these constraints. The range of clinical applications for which brain-computer interface technologies have been proposed includes neural recording and stimulation of anatomic and functional targets throughout the human brain $^{52,71-77}$. It seems unlikely that any single system or electrode type will be ideally suited to the full breadth of future needs in the domain of neural interfaces. Engineering tradeoffs must be made. Recognizing that a certain degree of application specificity is likely to persist in the field, we have chosen to prioritize safety and scalability with a view toward use in human clinical applications.

We believe that scalability and modularity are important features of neural interfaces designed for brain-computer interface applications. Scalability is a particularly important factor in designing neural interfaces with the intent to reach the large patient populations who stand to benefit from brain-computer interface technology. In considering applications such as paralysis, visual impairment, epilepsy, and some forms of cognitive impairment, the number of potential patients in the United States alone is in the many millions ${ }^{78-83}$. In particular, we believe it is important that the morbidity of the insertion procedure and the amount of brain tissue damaged or disrupted should be minimized and should not substantially increase as the number and density of implanted electrodes grow. Similarly, the total time required for implantation should scale in a favorable manner with respect to the number of electrodes. While several systems under development may support increasing channel count by orders of magnitude, it is not practical to lengthen surgical times by the same factors as doing so would mean prolonging a typical 1-hour operation to 10 hours or 100 hours or even longer. Here we have demonstrated an electrode array design and minimally invasive insertion technique that permit thousands of microelectrodes to be positioned on the cortical surface in a manner that scales favorably in terms of safety, electrode number and density, the spatial extent of the neural interface, and insertion time. The use of thinfilm surface microarrays shows promise for limiting neural tissue damage to undetectable levels while covering large areas of the cortical surface. The effective area of the microelectrode arrays described here is $0.48 \mathrm{~cm}^{2}$ per 529-channel module, and we have demonstrated that these arrays can be connected in a modular fashion and inserted simultaneously, at an effective rate exceeding $40 \mathrm{~s}$ per $\mathrm{cm}^{2}$ of cortical surface. Here, as proof of principle, we have demonstrated concurrent 
implantation over multiple functional areas of the cortical surface in both hemispheres. Considering the performance parameters demonstrated here, it becomes conceivable to envision deploying a thin-film-based neural interface over the majority of the accessible human neocortex.

Reversibility, in the sense of having the potential for explanting or replacement without damage to surrounding neural tissue, is an attractive property of candidate neural interfaces being considered for clinical use. Any implant may be expected to induce a certain degree of tissue encapsulation, but the anticipated tissue damage associated with removing multiple penetrating microelectrodes is greater than that associated with removing and replacing cortical surface arrays. Electrophysiologic performance from long-term implantation, as well as histologic data following removal of our chronically implanted arrays, will be forthcoming.

Effective neural decoding for brain-computer interfaces can be achieved using multiunit activity, local field potentials, or micro-electrocorticography, without relying on single-unit action potentials $^{22,84-91}$. For these reasons surface microarrays (micro-electrocorticography or $\mu \mathrm{ECoG}$ arrays) embody an appealing tradeoff between invasiveness and signal quality, and are attractive as an alternative or complement to arrays of electrodes that penetrate the cortical surface. Braincomputer interface methods derived from work in fundamental neuroscience have sometimes emphasized the action potential as the quantum of information transmission in the brain, and as the basis of systems for neural decoding. While these approaches have merit in animal models, they have less appeal in human clinical applications for which safety, effective function, and scalability are of primary importance. Microelectrode arrays that penetrate the brain facilitate single-cell recordings, at the cost of greater invasiveness, with varying degrees of brain tissue damage and long-term stability ${ }^{92-94}$. Microelectrode arrays that conform to the brain surface are considerably less invasive, more easily inserted and removed without damage to brain tissue, and potentially more stable in the long-term, but their greater distance from target neurons may limit single-cell communication. In practice, however, electrocorticography (ECoG) and microelectrocorticography $(\mu \mathrm{ECoG})$ have facilitated high temporal- and spatial-resolution recordings for effective use in in vivo applications ${ }^{56,64,65}$, including brain-computer interfaces. Several groups have used surface array techniques to correlate neural activity with motor function for the control of neural prostheses in physically impaired patients $7,67,68,95-98$. More recent work has demonstrated the utility of high-density ECoG in decoding neural representations of speech, enabling the articulation of words and short sentences ${ }^{6,69,99}$. These applications require the implanted arrays to be reliable and chronically stable. As a result, ultra-flexible arrays are now being considered as a promising alternative to more traditional ECoG grids, as they offer a more stable biotic-abiotic interface and in turn promote improved long-term performance. Recently developed thin-film $\mu \mathrm{ECoG}$ arrays have demonstrated the ability to conform to the brain surface safely for several months while reliably recording multi-unit and single-unit activity in certain anatomic settings ${ }^{53,57}$. These arrays can also serve as stimulation and biosensing platforms for multi-modal and closedloop brain-computer interface applications, though the high channel count and the extensive brain surface exposure typically required for these interfaces have made it challenging to exploit their potential in full using standard surgical techniques. The system described here represents a novel, minimally invasive approach to reliably implanting precisely this type of high-density array.

Specific electrode designs may be optimized for particular applications, such as single-unit recording or cortical stimulation to evoke a visual percept or motor response. Simultaneously optimizing for such diverse applications may not be feasible or necessary when designing neural interfaces for specific applications. However, certain functions are commonly required of braincomputer interfaces, and we demonstrate three key functions here: neural recording, neural 
decoding, and cortical stimulation. The incorporation of electrodes of different sizes on the same array partially facilitates this range of functions.

Multichannel $\mu \mathrm{ECoG}$ obtained during spontaneous activity is useful not only to evaluate the recording properties of the high-density microelectrode arrays, but also to understand and quantify the extent of correlation in neural activity across the cortical surface. The extent to which electrodes exhibit correlated activity depends on distance, electrode size, and the frequency band interrogated. High-frequency activity approaching the kilohertz range can exhibit relatively uncorrelated activity even at short range, consistent with the coexistence of nearby but relatively uncoupled neurons. On the other hand, cortical activity at the lower frequencies analyzed in traditional scalp EEG reflects bulk field activity and tends to be correlated across larger areas of the cortical surface. Quantifying these phenomena, as we begin to do here with high-density arrays capable of spanning large regions of cortical surface, is integral to designing surface arrays with optimal electrode geometry and spacing for use in neural prostheses that depend on accurate decoding of neural activity.

The rudimentary form of neural decoding we implement here reflects the robustness of electrocorticographic signals from multiple modalities, including somatosensation and vision, when viewed from the perspective of arrays positioned to span sufficiently large surface areas and with attention to functional neuroanatomy. The relative ease of accessing both hemispheres and multiple functional modalities (vision and somatic sensation) also emphasizes a key advantage to the minimally invasive "cranial micro-slit" approach facilitated by our arrays. More advanced forms of neural decoding will be described in forthcoming work, including some that employ contemporary approaches to parallel computation and machine learning techniques in optimizing the interpretation of array-based cortical signals.

Targeted neurostimulation is a key capability of closed-loop brain-computer interfaces, as well as neural prostheses for restoring functions such as vision. Here we characterize a safe operating regime for cortical stimulation from thin-film cortical surface microelectrodes, demonstrate the ability to access visual cortex in a minimally invasive fashion, electrophysiologically confirm array placement over functioning visual cortex, stimulate the cortical surface, and monitor stimulus-evoked cortical activity.

The system we describe here, the Layer 7 Cortical Interface, is a modular, scalable, minimally invasive brain-computer interface platform. The system is designed to deliver the benefits of high-density, high-channel-count neural interfaces to the millions of patients with neurologic disorders who stand to benefit from this technology. 


\section{Methods}

Array Fabrication and Characterization. Microelectrode arrays were fabricated on 8" silicon wafers using a spin-on polyimide. The fabrication process briefly comprised spin-coating, softbake, and vacuum cure of an approximately $10 \mu \mathrm{m}$ layer of polyimide; photolithographic patterning, deposition, and liftoff of $20 \mathrm{~nm} / 210 \mathrm{~nm} / 20 \mathrm{~nm} \mathrm{Ti} / \mathrm{Pt} / \mathrm{Ti}$ trace metal; $\mathrm{O}_{2}$ plasma treatment of the polyimide surface; spin-coating, soft-bake, and vacuum cure of an approximately $10 \mu \mathrm{m}$ layer of polyimide; hard mask deposition and patterning for polyimide outline and electrode site opening; polyimide etch and electrode surface exposure in $\mathrm{O}_{2} / \mathrm{CF}_{4}$ plasma; hard mask strip; photolithographic patterning, deposition, and liftoff of $20 \mathrm{~nm} / 20 \mathrm{~nm} / 500 \mathrm{~nm}$ of Ti/Pt/Au bond pad metallization; and $\mathrm{O}_{2}$ plasma post-clean of the polyimide surface. Following microfabrication, devices were released in deionized water, optically inspected for trace, electrode, and pad defects, dehydration baked, and thermocompression bonded to an organic interposer using a flip-chip tool.

The array pockets were laser cut from $25 \mu \mathrm{m}$ silicone adhesive-backed polyimide film using a low-power $3 \mathrm{~W}$ ultraviolet laser. The shape of the planar laser cut pattern was designed to correspond to the contour of the distal end of the microelectrode array with the addition of a 5.5 $\mathrm{mm}$ by $11.5 \mathrm{~mm}$ rectangular appendage protruding from the proximal edge. The rectangular appendage was folded under the adhesive side of the laser cut polyimide to occlude the adhesive and create the internal pocket feature of $5.5 \mathrm{~mm}$ width and $11.5 \mathrm{~mm}$ in length. The dimensions of the internal pocket feature were chosen to maintain a consistent lap joint width of $1.5 \mathrm{~mm}$ between the pocket and microelectrode array. Once the pocket had been laser cut and folded into its final configuration, it was then aligned with the $800 \mu \mathrm{m}$ alignment holes at the distal tip of the microelectrode array and compressed.

The microelectrode arrays are designed to be assembled into larger connected modules in a scalable fashion to achieve greater cortical coverage. Spacing and orientation were controlled during modular assembly with the assistance of alignment holes. The arrays were bonded by applying ISO 10993 biologically tested ultraviolet-curing cyanoacrylate to the overlapping regions of adjacent array modules.

Prior to assembly, bonded microelectrode array-interposer assemblies were optically inspected in bond, cable, and electrode areas, and a sampling of electrodes were characterized electrochemically. Electrochemical characterization was performed on a potentiostat (Wavedriver 100, Pine Research, Durham, North Carolina, United States of America) in a 3-electrode configuration (with $\mathrm{Ag} / \mathrm{AgCl}$ reference electrode and $\mathrm{Pt}$ coil counter electrode), and comprised cyclic voltammetry (CV) and electrochemical impedance spectroscopy (EIS) on at least one electrode per size in phosphate buffered saline (PBS) at $\mathrm{pH}$ 7.4. The $\mathrm{CV}$ measurements were performed (from 0 to $1.2 \mathrm{~V}$ to $-0.65 \mathrm{~V}$ to $0 \mathrm{~V}$ relative to the reference electrode) to confirm electrode surface identity using platinum oxidation and $\mathrm{Pt}-\mathrm{O}$ reduction peaks, hydrogen adsorption, and $\mathrm{H}_{2}$ oxidative desorption. In addition, $\mathrm{CV}$ measurements provide information on charge storage capacity and real surface area, and identify the water window. EIS measurements were performed from $10 \mathrm{~Hz}$ to $10 \mathrm{kHz}$ (on each electrode size) to confirm that $1 \mathrm{kHz}$ impedance and cutoff frequency are within expected ranges, and to provide references for later in vitro impedance mapping performed using the Intan chips in a two-electrode configuration. In vitro impedance mapping was performed in PBS on fully assembled devices (across all electrodes) at $100 \mathrm{~Hz}, 200 \mathrm{~Hz}, 500 \mathrm{~Hz}, 1,000 \mathrm{~Hz}, 2000 \mathrm{~Hz}$, and 5,000 Hz using the Intan chips in our custom 528-channel head stage. 
Minimally Invasive Surgical Implantation. In vivo testing of the minimally invasive surgical insertion technique and electrode array performance were performed in adult female Göttingen minipigs, selected for well characterized functional neuroanatomy as well as skull thickness comparable to that of adult humans. The study protocol was approved by the DaVinci Biomedical IACUC. Local anesthesia was achieved in the region of the skin incisions using intradermal lidocaine. General anesthesia was maintained with isoflurane at levels sufficient to produce analgesia without suppressing electrocorticographic activity, a balance that was facilitated by the minimally invasive nature of the procedure.

We developed a "cranial micro-slit" technique for array implantation. In order to insert each electrode array, a cranial incision was made using a customized 400-micron oscillating blade, at an entry angle tangential to the cortical surface. A 350-micron fiber-scope was then inserted through the cranial incision and used to visualize the dura, which was coagulated and cut under direct endoscopic vision. Endoscopy was similarly used to guide insertion of each electrode array into the subdural space. A $1.6 \mathrm{~mm}$ endoscope was used for intraoperative photography but was not used intracranially or introduced through the slit.

Electrode arrays were positioned subdurally on the cortical surface under simultaneous endoscopic and fluoroscopic guidance. Manipulation of each thin-film array was performed using a radiopaque stylet ("shim"). The stylet tip was designed to fit within a polyimide "pocket" on the reverse side of each array. Placement, depth, and angulation of cranial incisions and electrode arrays were also guided by fluoroscopy. Each stylet was removed following fluoroscopic confirmation of array position, leaving only the thin-film subdural microelectrode arrays in position on the cortical surface.

\section{Electrophysiology}

System Configuration and Recording Hardware. The customized neural recording and stimulation system is based on chips and controllers made by Intan Technologies (Los Angeles, California, United States of America). The custom amplifier printed circuit boards (PCBs) used to interface with the implanted electrode arrays each contained eight of the RHD2164 64-channel amplifier chips and one of the RHS2116 16-channel stimulator/amplifier chips, allowing for simultaneous recording from up to 528 channels and stimulation from up to 16 channels. In addition, each board allows for a hardware reference from one of 16 sites distributed across the array. The digitized data is transferred from the amplifier boards to an associated Intan Technologies 1,024-channel RHD controller or 126-channel RHS controller using low-voltage differential signaling (LVDS), where it is then stored on a USB-connected computer.

The amplifier boards are designed to allow each board to be easily coupled to any arrayinterposer assembly through the inclusion of an array of pogo pins that make contact with an associated pad on the array-interposer assembly, connecting each electrode site with an amplifier input. These two boards are aligned and held together by two plates with integrated alignment features placed on the outward-facing sides of the boards and screwed together. Additional protection of these electronics is provided by a custom, 3D-printed casing with strain-relief features for the electrode array and optional mounting braces to fix the entire assembly to the skull.

Recording Software and Data Pre-processing. The recording computers interface with either controller via a custom configuration of the Intan Technologies RHX Data Acquisition Software, which allows for real-time event-triggered averaging in addition to base functionality. 
The sampling rate for recording is set at $20 \mathrm{kHz}$ per channel, generating data at a rate over $2.5 \mathrm{~GB}$ per minute for each set of 1,024 channels. A $60 \mathrm{~Hz}$ notch filter is applied online during recording. For post-hoc analysis of local field potentials, unless otherwise specified, data is first downsampled to $5 \mathrm{kHz}$ using a Fourier method, then processed with a 5th-order Butterworth low-pass filter at $250 \mathrm{~Hz}$.

Free Recording of Spontaneous Cortical Activity. Example spectrograms are generated from data obtained at $20 \mathrm{kHz}$ per channel, where spectral density is computed for a temporal resolution of $50 \mathrm{~ms}$ and frequency resolution of $17.5 \mathrm{~Hz}$ using a Hann window.

To demonstrate spatial correlation between pairs of electrodes, downsampled stimulus-free neural data was separated into continuous 2 s segments. Within each segment, the Pearson correlation coefficient $r^{2}$ is computed for each pair of electrodes, and associated to the corresponding physical electrode distance. The average $r^{2}$ values across an array are pooled across 60 segments and averaged for each electrode distance.

Evoked Potentials. Somatosensory evoked potentials (SSEPs) are evoked by applying a periodic pressure stimulus at target locations in turn (right and left hindlimbs). Neural response waveforms are temporally aligned to the stimulus onset. SSEPs are then computed as the averaged time-aligned signals over 50 stimuli.

To elicit visual evoked potentials (VEP), the eyelid corresponding to the stimulated retina is retracted temporarily while periodic $50 \mathrm{~ms}$ flashes are generated at $1 \mathrm{~Hz}$ from an array of white light-emitting diodes. Neural response waveforms are temporally aligned to the stimulus onset. VEPs are calculated as the time-aligned averaged signals over 150 trials.

Cortical Stimulation. Electrical stimulation at the cortical surface is applied at one of the $200 \mu \mathrm{m}$ electrodes, controlled by the Intan Technologies RHS controller and RHX software. Charge-balanced, biphasic, cathodic-first, $200 \mu$ s pulses of $100 \mu \mathrm{A}$ peak current are delivered at $0.25 \mathrm{~Hz}$. The evoked potentials are recorded over a series of trials. During analysis, for each trial and electrode, $35 \mathrm{~ms}$ segments after variable delay $(0,2,5,10,15,20,25,30,50 \mathrm{~ms})$ post-stimulus are first Fourier-transformed, then integrated for spectral power in the high-gamma band (70-150 $\mathrm{Hz}$ ) and averaged over 25 trials.

Neural Decoding. Single- and multi-channel, one-shot, binary classification decoding efficacy is demonstrated with a template matching approach. Recording segments of $1 \mathrm{~s}$ duration are classified as either an evoked potential or spontaneous activity. VEPs obtained by photostimulation of the left eye and right eye are taken as templates, and $r^{2}$ is computed between the template and recording segment. VEPs are computed over 150 trials as described previously, and template matching is tested over $20 \mathrm{VEP}$ trials and 20 segments of spontaneous activity. The receiver operating characteristic (ROC) curve and accuracy are then calculated by varying threshold $r^{2}$ value. Whole-array decoding operates similarly with the single-channel template replaced by an unweighted concatenation of all single-channel templates. Curves representing the average ROC performance were obtained by reshuffling and resampling the training and testing trials 20 times in Monte Carlo fashion. 


\section{Author Contributions}

Study conception and design: BIR, EH, MH, DP, AP, MV, MM. Data collection: BIR, EH, MH, DP, AP, KG. Interpretation of results: BIR, EH, MH, DP, AP, MV, MM, MM, TH, VT, KG. Manuscript preparation: BIR, EH, MH, DP, MV, AP, MM, MM. All authors reviewed the results and approved this version of the manuscript.

\section{Acknowledgments}

The authors gratefully acknowledge the assistance of Craig H. Mermel, Jason L. Chen, Jonathan Steinberger, and Rony Abovitz for critical review of this manuscript. The authors also gratefully acknowledge the artistic and logistical assistance of Helen Melville.

Correspondence and requests for materials should be addressed to Benjamin I. Rapoport at ben@precisionneuro.io. 


\section{References}

1. Bouton, C. E. et al. Restoring cortical control of functional movement in a human with quadriplegia. Nature 533, 247-250 (2016).

2. Kim, S.-P., Simeral, J. D., Hochberg, L. R., Donoghue, J. P. \& Black, M. J. Neural control of computer cursor velocity by decoding motor cortical spiking activity in humans with tetraplegia. J. Neural Eng. 5, 455-476 (2008).

3. Pandarinath, C. et al. High performance communication by people with paralysis using an intracortical brain-computer interface. eLife 6, e18554 (2017).

4. Simeral, J. D., Kim, S.-P., Black, M. J., Donoghue, J. P. \& Hochberg, L. R. Neural control of cursor trajectory and click by a human with tetraplegia 1000 days after implant of an intracortical microelectrode array. J. Neural Eng. 8, 025027 (2011).

5. Gilja, V. et al. Clinical translation of a high-performance neural prosthesis. Nat. Med. 21, 1142-1145 (2015).

6. Moses, D. A. et al. Neuroprosthesis for Decoding Speech in a Paralyzed Person with Anarthria. N. Engl. J. Med. 385, 217-227 (2021).

7. Wang, W. et al. An Electrocorticographic Brain Interface in an Individual with Tetraplegia. PLoS ONE 8, e55344 (2013).

8. Hochberg, L. R. et al. Neuronal ensemble control of prosthetic devices by a human with tetraplegia. Nature 442, 164-171 (2006).

9. Velliste, M., Perel, S., Spalding, M. C., Whitford, A. S. \& Schwartz, A. B. Cortical control of a prosthetic arm for self-feeding. Nature 453, 1098-1101 (2008).

10. Schwartz, A. B., Cui, X. T., Weber, D. J. \& Moran, D. W. Brain-Controlled Interfaces: Movement Restoration with Neural Prosthetics. Neuron 52, 205-220 (2006).

11. Collinger, J. L. et al. High-performance neuroprosthetic control by an individual with tetraplegia. The Lancet 381, 557-564 (2013).

12. Wang, W. et al. Human motor cortical activity recorded with Micro-ECoG electrodes, during individual finger movements. in 2009 Annual International Conference of the IEEE Engineering in Medicine and Biology Society 586-589 (IEEE, 2009). doi:10.1109/IEMBS.2009.5333704.

13. Wessberg, J. et al. Real-time prediction of hand trajectory by ensembles of cortical neurons in primates. Nature 408, 361-365 (2000).

14. Carmena, J. M. et al. Learning to Control a Brain-Machine Interface for Reaching and Grasping by Primates. PLoS Biol. 1, e42 (2003).

15. Lebedev, M. A. \& Nicolelis, M. A. L. Brain-machine interfaces: past, present and future. Trends Neurosci. 29, 536-546 (2006).

16. Fraser, G. W., Chase, S. M., Whitford, A. \& Schwartz, A. B. Control of a brain-computer interface without spike sorting. J. Neural Eng. 6, 055004 (2009).

17. Gilja, V. et al. A high-performance neural prosthesis enabled by control algorithm design. Nat. Neurosci. 15, 1752-1757 (2012).

18. Pandarinath, C. et al. Inferring single-trial neural population dynamics using sequential autoencoders. Nat. Methods 15, 805-815 (2018).

19. Collinger, J. L., Gaunt, R. A. \& Schwartz, A. B. Progress towards restoring upper limb movement and sensation through intracortical brain-computer interfaces. Curr. Opin. Biomed. Eng. 8, 84-92 (2018).

20. Musk, E. \& Neuralink. An integrated brain-machine interface platform with thousands of 
channels. http://biorxiv.org/lookup/doi/10.1101/703801 (2019) doi:10.1101/703801.

21. Sahasrabuddhe, K. et al. The Argo: A 65,536 channel recording system for high density neural recording in vivo. http://biorxiv.org/lookup/doi/10.1101/2020.07.17.209403 (2020) doi:10.1101/2020.07.17.209403.

22. Schalk, G. et al. Decoding two-dimensional movement trajectories using electrocorticographic signals in humans. J. Neural Eng. 4, 264-275 (2007).

23. Wissel, T. et al. Hidden Markov model and support vector machine based decoding of finger movements using electrocorticography. J. Neural Eng. 10, 056020 (2013).

24. Moses, D. A., Leonard, M. K., Makin, J. G. \& Chang, E. F. Real-time decoding of questionand-answer speech dialogue using human cortical activity. Nat. Commun. 10, 3096 (2019).

25. Sun, P., Anumanchipalli, G. K. \& Chang, E. F. Brain2Char: a deep architecture for decoding text from brain recordings. J. Neural Eng. 17, 066015 (2020).

26. Yu, B. M. et al. Mixture of Trajectory Models for Neural Decoding of Goal-Directed Movements. J. Neurophysiol. 97, 3763-3780 (2007).

27. Kemere, C., Shenoy, K. V. \& Meng, T. H. Model-Based Neural Decoding of Reaching Movements: A Maximum Likelihood Approach. IEEE Trans. Biomed. Eng. 51, 925-932 (2004).

28. Wilson, G. H. et al. Decoding spoken English from intracortical electrode arrays in dorsal precentral gyrus. J. Neural Eng. 17, 066007 (2020).

29. Harrison, R. R. \& Charles, C. A low-power low-noise cmos for amplifier neural recording applications. IEEE J. Solid-State Circuits 38, 958-965 (2003).

30. Harrison, R. R. et al. A Low-Power Integrated Circuit for a Wireless 100-Electrode Neural Recording System. IEEE J. Solid-State Circuits 42, 123-133 (2007).

31. Sarpeshkar, R. et al. Low-Power Circuits for Brain-Machine Interfaces. IEEE Trans. Biomed. Circuits Syst. 2, 173-183 (2008).

32. Mora Lopez, C. et al. A Neural Probe With Up to 966 Electrodes and Up to 384 Configurable Channels in $0.13 \$ \backslash \mathrm{mu} \$ \mathrm{~m}$ SOI CMOS. IEEE Trans. Biomed. Circuits Syst. 11, 510-522 (2017).

33. Wang, S. et al. A Compact Quad-Shank CMOS Neural Probe With 5,120 Addressable Recording Sites and 384 Fully Differential Parallel Channels. IEEE Trans. Biomed. Circuits Syst. 13, 1625-1634 (2019).

34. Biederman, W. et al. A $4.78 \mathrm{~mm} 2$ Fully-Integrated Neuromodulation SoC Combining 64 Acquisition Channels With Digital Compression and Simultaneous Dual Stimulation. IEEE J. Solid-State Circuits 50, 1038-1047 (2015).

35. Yoon, D.-Y. et al. A 1024-Channel Simultaneous Recording Neural SoC with Stimulation and Real-Time Spike Detection. in 2021 Symposium on VLSI Circuits 1-2 (IEEE, 2021). doi:10.23919/VLSICircuits52068.2021.9492480.

36. Johnson, B. C. et al. An implantable $700 \mu \mathrm{W}$ 64-channel neuromodulation IC for simultaneous recording and stimulation with rapid artifact recovery. in 2017 Symposium on VLSI Circuits C48-C49 (IEEE, 2017). doi:10.23919/VLSIC.2017.8008543.

37. Lee, J. et al. Neural recording and stimulation using wireless networks of microimplants. Nat. Electron. 4, 604-614 (2021).

38. Ha, S. et al. Silicon-Integrated High-Density Electrocortical Interfaces. Proc. IEEE 105, $11-$ 33 (2017).

39. Ahmadi, N. et al. Towards a Distributed, Chronically-Implantable Neural Interface. in 2019 9th International IEEE/EMBS Conference on Neural Engineering (NER) 719-724 (IEEE, 
2019). doi:10.1109/NER.2019.8716998.

40. Seo, D. et al. Wireless Recording in the Peripheral Nervous System with Ultrasonic Neural Dust. Neuron 91, 529-539 (2016).

41. O’Leary, G., Groppe, D. M., Valiante, T. A., Verma, N. \& Genov, R. NURIP: Neural Interface Processor for Brain-State Classification and Programmable-Waveform Neurostimulation. IEEE J. Solid-State Circuits 53, 3150-3162 (2018).

42. Cogan, S. F. Neural Stimulation and Recording Electrodes. Annu. Rev. Biomed. Eng. 10, 275-309 (2008).

43. Wellman, S. M. et al. A Materials Roadmap to Functional Neural Interface Design. Adv. Funct. Mater. 28, 1701269 (2018).

44. Ordonez, J. S., Boehler, C., Schuettler, M. \& Stieglitz, T. Improved polyimide thin-film electrodes for neural implants. in 2012 Annual International Conference of the IEEE Engineering in Medicine and Biology Society 5134-5137 (IEEE, 2012). doi:10.1109/EMBC.2012.6347149.

45. Vomero, M. et al. Incorporation of Silicon Carbide and Diamond-Like Carbon as Adhesion Promoters Improves In Vitro and In Vivo Stability of Thin-Film Glassy Carbon Electrocorticography Arrays. Adv. Biosyst. 2, 1700081 (2018).

46. Deku, F. et al. Amorphous silicon carbide ultramicroelectrode arrays for neural stimulation and recording. J. Neural Eng. 15, 016007 (2018).

47. Li et al. Ultra-Long-Term Reliable Encapsulation Using an Atomic Layer Deposited HfO2/A12O3/HfO2 Triple-Interlayer for Biomedical Implants. Coatings 9, 579 (2019).

48. Jeong, J. et al. Conformal Hermetic Sealing of Wireless Microelectronic Implantable Chiplets by Multilayered Atomic Layer Deposition (ALD). Adv. Funct. Mater. 29, 1806440 (2019).

49. Lamont, C. et al. Silicone encapsulation of thin-film $\mathrm{SiO}_{\mathrm{x}}, \mathrm{SiO} \times \mathrm{N}_{\text {y }}$ and $\mathrm{SiC}$ for modern electronic medical implants: a comparative long-term ageing study. J. Neural Eng. (2021) doi:10.1088/1741-2552/abf0d6.

50. Cook, M. J. et al. Prediction of seizure likelihood with a long-term, implanted seizure advisory system in patients with drug-resistant epilepsy: a first-in-man study. Lancet Neurol. 12, 563-571 (2013).

51. Smalley, E. The business of brain-computer interfaces. Nat. Biotechnol. 37, 978-982 (2019).

52. Lebedev, M. A. \& Nicolelis, M. A. L. Brain-Machine Interfaces: From Basic Science to Neuroprostheses and Neurorehabilitation. Physiol. Rev. 97, 767-837 (2017).

53. Vomero, M. et al. Conformable polyimide-based $\mu$ ECoGs: Bringing the electrodes closer to the signal source. Biomaterials 255, 120178 (2020).

54. Bockhorst, T. et al. Synchrony surfacing: Epicortical recording of correlated action potentials. Eur. J. Neurosci. 48, 3583-3596 (2018).

55. Minev, I. R. et al. Electronic dura mater for long-term multimodal neural interfaces. Science 347, 159-163 (2015).

56. Viventi, J. et al. Flexible, foldable, actively multiplexed, high-density electrode array for mapping brain activity in vivo. Nat. Neurosci. 14, 1599-1605 (2011).

57. Khodagholy, D. et al. NeuroGrid: recording action potentials from the surface of the brain. Nat. Neurosci. 18, 310-315 (2015).

58. Khodagholy, D. et al. Highly Conformable Conducting Polymer Electrodes for In Vivo Recordings. Adv. Mater. 23, H268-H272 (2011).

59. Chung, J. E. et al. High-Density, Long-Lasting, and Multi-region Electrophysiological 
Recordings Using Polymer Electrode Arrays. Neuron 101, 21-31.e5 (2019).

60. Hirschberg, A. W. et al. Development of an anatomically conformal parylene neural probe array for multi-region hippocampal recordings. in 2017 IEEE 30th International Conference on Micro Electro Mechanical Systems (MEMS) 129-132 (IEEE, 2017). doi:10.1109/MEMSYS.2017.7863357.

61. Hara, S. A. et al. Long-term stability of intracortical recordings using perforated and arrayed Parylene sheath electrodes. J. Neural Eng. 13, 066020 (2016).

62. Kim, B. J. et al. 3D Parylene sheath neural probe for chronic recordings. J. Neural Eng. 10, 045002 (2013).

63. Chiang, C.-H. et al. Flexible, high-resolution thin-film electrodes for human and animal neural research. J. Neural Eng. 18, 045009 (2021).

64. Kim, D.-H., Ghaffari, R., Lu, N. \& Rogers, J. A. Flexible and Stretchable Electronics for Biointegrated Devices. Annu. Rev. Biomed. Eng. 14, 113-128 (2012).

65. Rubehn, B., Bosman, C., Oostenveld, R., Fries, P. \& Stieglitz, T. A MEMS-based flexible multichannel ECoG-electrode array. J. Neural Eng. 6, 036003 (2009).

66. Salari, E. et al. Classification of Articulator Movements and Movement Direction from Sensorimotor Cortex Activity. Sci. Rep. 9, 14165 (2019).

67. Schalk, G. et al. Two-dimensional movement control using electrocorticographic signals in humans. J. Neural Eng. 5, 75-84 (2008).

68. Pistohl, T., Ball, T., Schulze-Bonhage, A., Aertsen, A. \& Mehring, C. Prediction of arm movement trajectories from ECoG-recordings in humans. J. Neurosci. Methods 167, 105114 (2008).

69. Anumanchipalli, G. K., Chartier, J. \& Chang, E. F. Speech synthesis from neural decoding of spoken sentences. Nature 568, 493-498 (2019).

70. Angrick, M. et al. Speech synthesis from ECoG using densely connected 3D convolutional neural networks. J. Neural Eng. 16, 036019 (2019).

71. Brindley, G. S. \& Lewin, W. S. The sensations produced by electrical stimulation of the visual cortex. J. Physiol. 196, 479-493 (1968).

72. Dobelle, W. H., Mladejovsky, M. G. \& Girvin, J. P. Artificial Vision for the Blind: Electrical Stimulation of Visual Cortex Offers Hope for a Functional Prosthesis. Science 183, 440-444 (1974).

73. Dobelle, W. H. et al. Artificial Vision for the Blind by Electrical Stimulation of the Visual Cortex. Neurosurgery 5, 521-527 (1979).

74. Eddington, D. K. Speech discrimination in deaf subjects with cochlear implants. J. Acoust. Soc. Am. 68, 885-891 (1980).

75. Wilson, B. S. \& Dorman, M. F. Cochlear implants: A remarkable past and a brilliant future. Hear. Res. 242, 3-21 (2008).

76. Weiland, J. D., Liu, W. \& Humayun, M. S. Retinal Prosthesis. Annu. Rev. Biomed. Eng. 7, 361-401 (2005).

77. Lewis, P. M., Ackland, H. M., Lowery, A. J. \& Rosenfeld, J. V. Restoration of vision in blind individuals using bionic devices: A review with a focus on cortical visual prostheses. Brain Res. 1595, 51-73 (2015).

78. Kang, Y. et al. Epidemiology of worldwide spinal cord injury: a literature review. $J$. Neurorestoratology Volume 6, 1-9 (2017).

79. Lo, J., Chan, L. \& Flynn, S. A Systematic Review of the Incidence, Prevalence, Costs, and Activity and Work Limitations of Amputation, Osteoarthritis, Rheumatoid Arthritis, Back 
Pain, Multiple Sclerosis, Spinal Cord Injury, Stroke, and Traumatic Brain Injury in the United States: A 2019 Update. Arch. Phys. Med. Rehabil. 102, 115-131 (2021).

80. Katan, M. \& Luft, A. Global Burden of Stroke. Semin. Neurol. 38, 208-211 (2018).

81. Zack, M. M. \& Kobau, R. National and State Estimates of the Numbers of Adults and Children with Active Epilepsy — United States, 2015. MMWR Morb. Mortal. Wkly. Rep. 66, 821-825 (2017).

82. Chan, T., Friedman, D. S., Bradley, C. \& Massof, R. Estimates of Incidence and Prevalence of Visual Impairment, Low Vision, and Blindness in the United States. JAMA Ophthalmol. 136, 12 (2018).

83. Matthews, K. A. et al. Racial and ethnic estimates of Alzheimer's disease and related dementias in the United States (2015-2060) in adults aged $\geq 65$ years. Alzheimers Dement. 15, 17-24 (2019).

84. Pesaran, B., Pezaris, J. S., Sahani, M., Mitra, P. P. \& Andersen, R. A. Temporal structure in neuronal activity during working memory in macaque parietal cortex. Nat. Neurosci. 5, 805811 (2002).

85. Andersen, R. A., Musallam, S. \& Pesaran, B. Selecting the signals for a brain-machine interface. Curr. Opin. Neurobiol. 14, 720-726 (2004).

86. Scherberger, H., Jarvis, M. R. \& Andersen, R. A. Cortical Local Field Potential Encodes Movement Intentions in the Posterior Parietal Cortex. Neuron 46, 347-354 (2005).

87. Jun Zhuang, Truccolo, W., Vargas-Irwin, C. \& Donoghue, J. P. Decoding 3-D Reach and Grasp Kinematics From High-Frequency Local Field Potentials in Primate Primary Motor Cortex. IEEE Trans. Biomed. Eng. 57, 1774-1784 (2010).

88. Bansal, A. K., Truccolo, W., Vargas-Irwin, C. E. \& Donoghue, J. P. Decoding 3D reach and grasp from hybrid signals in motor and premotor cortices: spikes, multiunit activity, and local field potentials. J. Neurophysiol. 107, 1337-1355 (2012).

89. Bansal, A. K., Vargas-Irwin, C. E., Truccolo, W. \& Donoghue, J. P. Relationships among low-frequency local field potentials, spiking activity, and three-dimensional reach and grasp kinematics in primary motor and ventral premotor cortices. J. Neurophysiol. 105, 1603-1619 (2011).

90. Markowitz, D. A., Wong, Y. T., Gray, C. M. \& Pesaran, B. Optimizing the Decoding of Movement Goals from Local Field Potentials in Macaque Cortex. J. Neurosci. 31, 1841218422 (2011).

91. Volkova, K., Lebedev, M. A., Kaplan, A. \& Ossadtchi, A. Decoding Movement From Electrocorticographic Activity: A Review. Front. Neuroinformatics 13, 74 (2019).

92. Polikov, V. S., Tresco, P. A. \& Reichert, W. M. Response of brain tissue to chronically implanted neural electrodes. J. Neurosci. Methods 148, 1-18 (2005).

93. Biran, R., Martin, D. C. \& Tresco, P. A. Neuronal cell loss accompanies the brain tissue response to chronically implanted silicon microelectrode arrays. Exp. Neurol. 195, 115-126 (2005).

94. Winslow, B. D. \& Tresco, P. A. Quantitative analysis of the tissue response to chronically implanted microwire electrodes in rat cortex. Biomaterials 31, 1558-1567 (2010).

95. Branco, M. P. et al. Decoding hand gestures from primary somatosensory cortex using highdensity ECoG. NeuroImage 147, 130-142 (2017).

96. Nakanishi, Y. et al. Prediction of Three-Dimensional Arm Trajectories Based on ECoG Signals Recorded from Human Sensorimotor Cortex. PLoS ONE 8, e72085 (2013).

97. Pistohl, T. et al. Grasp Detection from Human ECoG during Natural Reach-to-Grasp 
Movements. PLoS ONE 8, e54658 (2013).

98. Pistohl, T., Schulze-Bonhage, A., Aertsen, A., Mehring, C. \& Ball, T. Decoding natural grasp types from human ECoG. NeuroImage 59, 248-260 (2012).

99. Makin, J. G., Moses, D. A. \& Chang, E. F. Machine translation of cortical activity to text with an encoder-decoder framework. Nat. Neurosci. 23, 575-582 (2020). 